\title{
Especies de garrapatas en un área urbana protegida de la Ciudad Autónoma de Buenos Aires
}

\author{
Ticks species in a protected area of the Autonomous City of Buenos Aires \\ Sandra González ${ }^{1}$, Luciana Graciano², Luciana Garaglia², Gabriel Cicuttin ${ }^{3}$, \\ Edgardo Marcos², Osvaldo Degregorio ${ }^{2}$
}

\section{Resumen}

\begin{abstract}
El presente estudio se realizó con el objetivo de determinar la presencia de garrapatas en la Reserva Ecológica Ciudad Universitaria - Costanera Norte, área natural protegida de la $\mathrm{CABA}$, lindante con un área urbana con alto tránsito de personas. Entre agosto de 2017 y julio de 2018 se recolectaron 829 Ixodes auritulus (larvas, ninfas, adultos) y una ninfa de Rhipicephalus sanguineus sensu lato de la vegetación en tres puntos muestreados mensualmente.
\end{abstract}

Palabras clave: garrapatas; Ixodes auritulus; área natural protegida

\section{AbSTRACT}

The present study aimed to determine the presence of ticks in the Ecological Reserve Ciudad Universitaria - Costanera Norte, protected natural area of the CABA, close to a highly trafficked urban area. In the period August 2017 to July 2018, 829 Ixodes auritulus (larvae, nymphs, adults) and a nymph of Rhipicephalus sanguineus sensu lato were collected from the vegetation in three sample sites on a monthly interval.

Keys words: ticks; Ixodes auritulus; protected natural area

${ }^{1}$ Cátedra de Bioestadística, Facultad de Ciencias Veterinarias, Universidad de Buenos Aires, Buenos Aires, Argentina

${ }^{2}$ Cátedra de Salud Pública, Facultad de Ciencias Veterinarias, Universidad de Buenos Aires, Argentina

${ }^{3}$ Instituto de Zoonosis Luis Pasteur, Buenos Aires, Argentina

${ }^{4}$ E-mail: odegre@fvet.uba.ar

Recibido: 26 de febrero de 2019

Aceptado para publicación: 26 de octubre de 2019 
INTRODUCCIÓN

Las garrapatas (Acari, Ixodida) son ectoparásitos hematófagos de vertebrados (con excepción de los peces), con capacidad de provocar parálisis, toxicosis, alergias y ser vectores de diversos microorganismos (virus, bacterias y protozoos) que pueden provocar enfermedades de importancia en medicina humana y veterinaria (Cicuttin, 2016; Cicuttin et al., 2017a; Nava et al., 2017).

Las áreas urbanas protegidas son zonas ecológicamente significativas que conservan la biodiversidad y se encuentran dentro de grandes centros urbanos o limitan con ellos. Son consideradas esenciales para la conservación de la diversidad biológica y el mantenimiento de ambientes saludables para las personas. El hombre y los animales interactúan sobre estas interfases (silvestresurbanas) dando lugar a numerosas oportunidades para la transmisión de enfermedades zoonóticas (Soler-Tovar et al., 2010; Danta Torres et al., 2012; Marcos, 2013; Cicuttin, 2016; Cicuttin et al., 2017; Sirolli et al., 2018). Las características de estas interfases, como la cercanía entre los ambientes urbanos y silvestres, y el posible tránsito de animales domésticos y sinantrópicos entre ambas las transforman en interesantes sistemas de estudio de las garrapatas como potenciales vectores de zoonosis (Parola y Raoult, 2001; Päpffle et al., 2015).

En Argentina se encuentran numerosas especies de garrapatas duras (familia Ixodidae) de los géneros Amblyomma (23 especies), Dermacentor (1), Haemaphysalis (2), Ixodes (10) y Rhipicephalus (2). Estudios previos en Ciudad Autónoma de Buenos Aires (CABA) indican la presencia de cuatro especies: Rhipicephalus sanguineus sensu lato, Amblyomma aureolatum, Amblyomma triste e Ixodes auritulus, habiendo sido encontradas las tres últimas exclusivamente en la Reserva Ecológica Costanera Sur (RECS) (Parola y Raoult, 2001; Cicuttin et al., 2017; Nava et al., 2017). El objetivo del presente estudio fue determinar la presencia de especies de garrapatas en la Reserva Ecológica Ciudad Universitaria Costanera Norte (RECU-CN), área urbana protegida de la CABA.

\section{Materiales y Métodos}

La RECU-CN $\left(34^{\circ} 32223.543 \mathrm{~S}\right.$; $58^{\circ} 26238.623 \mathrm{O}$ ) es un área urbana protegida al norte de la CABA, a orillas del Río de la Plata (Argentina). Sus límites son la Ciudad Universitaria (campus de la Universidad de Buenos Aires), la desembocadura del arroyo White, el Río de la Plata y el Parque de la Memoria. Su superficie total es de 23 hectáreas. Se asienta sobre terrenos ganados al Río de la Plata, mediante rellenos antrópicos realizados en las décadas del 60 y 70 . El área posee un clima de tipo templado húmedo con precipitaciones durante todo el año. Según la vegetación y cobertura dominante se definen cuatro ambientes: humedal, bosque y arboleda, pastizal y superficie antrópica. Se han identificado 522 especies de animales y 234 de plantas (Sirolli et al., 2018). En el área lindante a la reserva se desarrollan las actividades del Centro Experimental de Permacultura, Desarrollo Sustentable y Educación Ambiental, denominado Velatropa (Eco Aldea).

Las garrapatas se colectaron mensualmente entre agosto de 2017 y julio de 2018 , mediante el método bandera o «dragging» de la vegetación, en su fase de vida libre. El método consiste en arrastrar un paño de algodón blanco de aproximadamente $1.0 \mathrm{~m} \mathrm{x}$ 1.5 m (Nava et al., 2011; Cicuttin et al., 2017). Se definieron tres sitios para los muestreos y fueron recorridos por $30 \mathrm{minu}-$ tos cada uno, revisando cada 10 metros el paño y removiendo los ejemplares capturados. Las garrapatas obtenidas fueron conservadas en alcohol 70\%. La clasificación morfológica se realizó mediante lupa binocular estereoscópica y utilizando claves taxonómicas (Nava et al., 2017). 


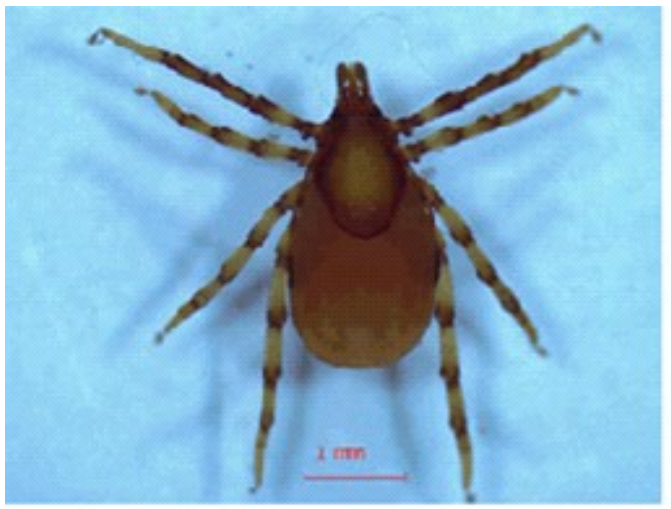

(a)

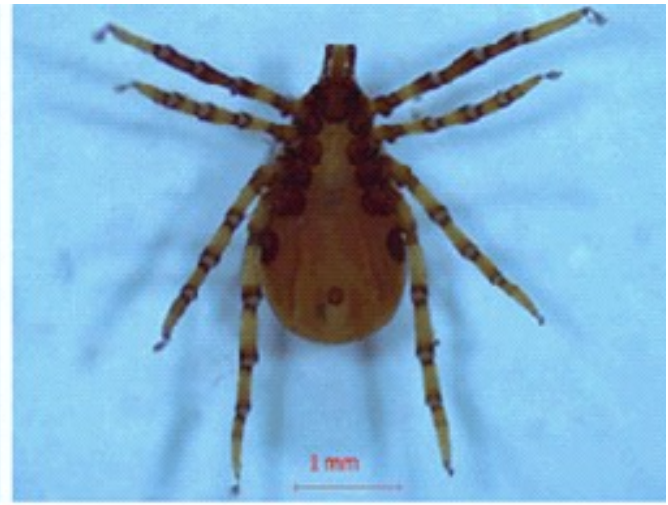

(b)

Figura 1. Larvas y ninfa de Ixodes auritulus colectados en un área urbana de la Ciudad Autónoma de Buenos Airestónoma de Buenos Aires. (a) Ninfa vista desde dorsal; (b) Ninfa vista desde ventral

\section{Resultados}

Se colectaron 830 garrapatas de la vegetación. La identificación taxonómica determinó que 829 garrapatas eran de la especie Ixodes auritulus (776 larvas, 50 ninfas y 3 hembras) y una ninfa de la especie Rhipicephalus sanguineus s. 1 (Figura 1), esta última colectada en el mes de enero. La Figura 2 presenta la distribución estacional de I. auritulus, observándose la presencia de larvas y ninfas durante todo el año, con picos de abundancia para ambos estadios en los meses fríos. En el caso de las larvas en mayo y junio, y para las ninfas en mayo y julio. Los adultos fueron encontrados en los meses de enero, febrero y junio. Se hace notar que en el mes de abril no se pudo realizar el muestreo debido a las condiciones climáticas adversas.

\section{Discusión}

El presente estudio comprobó la presencia estable de Ixodes auritulus en la RECU$\mathrm{CN}$. Esta garrapata es un parásito de aves con distribución mundial, aunque se considera que podría tratarse de un complejo de especies (Nava et al., 2017). En Sudamérica presenta una distribución focal en Chile, Brasil, Perú, Uruguay y Argentina. En este último país existe escasa información respecto de esta especie, siendo registrada en Patagonia, en la zona del bajo Delta del Río de Paraná y en la RECS de CABA, donde también se considera establecida, con una distribución estacional similar al presente estudio (Guglielmone y Nava, 2005; Flores et al., 2014; Cicuttin et al., 2017; Nava et al., 2017). No se conocen registros de picaduras en humanos, pero son reconocidas por su rol 


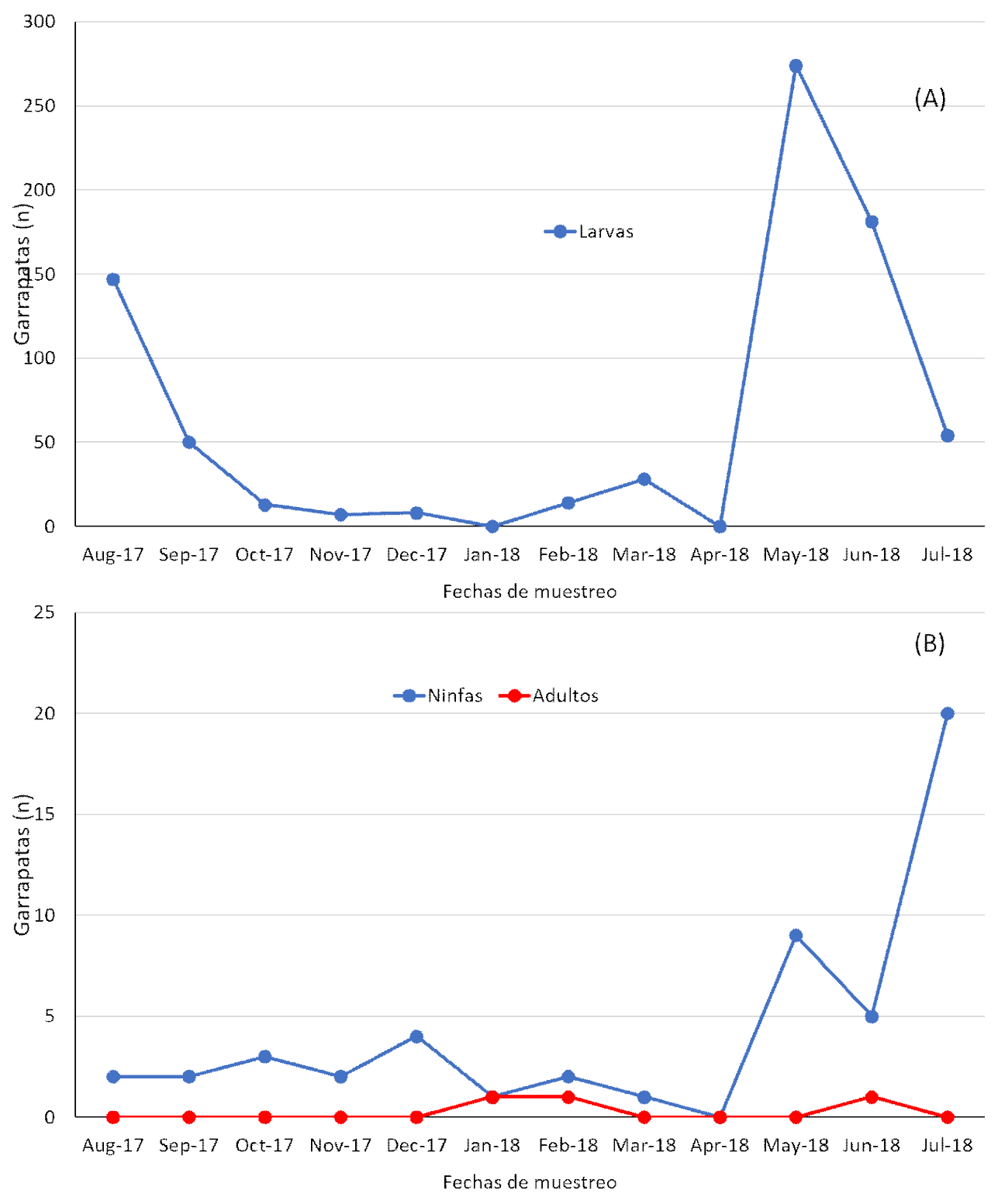

Figura 2. Distribución estacional de garrapatas Ixodes auritulus recolectadas de la vegetación de la Reserva Ecológica Ciudad Universitaria - Costanera Norte (RECU-CN), Buenos Aires, Argentina entre agosto de 2017 y julio de 2018. (A) Larvas; (B) Ninfas y adultos

en el mantenimiento del ciclo de Borrelia burgdorferi sensu lato en aves de América del Norte (Cicuttin, 2016; Cicuttin et al., 2017a). En Argentina, Cicuttin et al. (2017b) detectaron Borrelia sp del grupo de Borrelia burgdorferi s. 1. en esta especie de garrapata.
Rhipicephalus sanguineus s.1. presenta una amplia distribución mundial, siendo el perro el principal hospedador, aunque ha sido hallada ocasionalmente sobre otros mamíferos, incluidos seres humanos. La presencia de $R$. sanguineus $\mathrm{s}$. 1. es de esperarse, dada la pre- 
ferencia de esta especie por áreas urbanas, especialmente en época estival en CABA y la presencia de perros vagabundos en RECUCN (Guglielmone y Nava, 2005; Cicuttin 2016). $R$. sanguineus es vector de numerosos patógenos de importancia médica y veterinaria. En Argentina, se ha detectado Rickettsia massiliae (agente de rickettsiosis humana) en $R$. sanguineus s. 1. de CABA, Bahía Blanca y Mendoza (Cicuttin, 2014).

El área estudiada presenta la particularidad de contar con una gran afluencia de personas, algunas provenientes de Ciudad Universitaria y otras que hacen uso recreativo de la misma (paseos, caminatas o pesca). Se ha identificado, asimismo, dos asentamientos permanentes con cinco individuos como máximo y otros asentamientos temporales, con la presencia de caninos y felinos domésticos en ambos casos (Sirolli et al., 2018). Los resultados del estudio permiten plantear la necesidad de continuar con el estudio de garrapatas y patógenos asociadas en la RECU-CN de CABA, sobre todo considerando la importancia de las enfermedades transmitidas por garrapatas.

\section{Literatura Citada}

1. Cicuttin G, De Salvo M, Nava S. 2017a. Especies de garrapatas duras en un área natural protegida de la Ciudad Autónoma de Buenos Aires. Rev Argent Salud Pública 8: 7-12.

2. Cicuttin G De Salvo M, Nava S. 2017 . Genoespecies de Borrelia presentes en garrapatas duras (Acari: Ixodidae) colectadas en un área urbana protegida de la Ciudad Autónoma de Buenos Aires. En: Simposio Internacional sobre Enfermedades Desatendidas. Buenos Aires.

3. Cicuttin GL. 2016. Presencia de garrapatas duras presentes en la Ciudad Autónoma de Buenos Aires y su importancia para la Salud Pública. Rev Argent Zoonosis Enf Infec Emerg 11: 5-9.
4. Cicuttin GL. 2014. Rickettsiosis urbanas: Rickettsia massiliae. En: Farjat JB, Enría D, Martino P, Rosenzvit M, Seijo A (eds). Temas de Zoonosis VI. Buenos Aires: Asociación Argentina de Zoonosis. p 281-286.

5. Danta-Torres F, Chomel BB, Otranto D. 2012. Ticks and tick-borne diseases: a One Health perspective. Trends Parasitol 28: 437-446. doi: 10.1016/ j.pt.2012.07.003

6. Flores FS, Nava S, Batallán G, Tauro LB, Contigiani MS, Diaz LA, Guglielmone AA. 2014. Ticks (Acari: Ixodidae) on wild birds in north-central Argentina. Ticks Tick-Borne Dis 5: 715-721.

7. Guglielmone A, Nava S. 2005. Las garrapatas de la familia Argaside y de los géneros Dermacentor, Haemaphysalis, Ixodes y Rhipicephalus de la Argentina. RIA 34: 123-141.

8. Marcos ER. 2013. El concepto una salud como integrador de la interfase humano-animal-ambiente, frente a las enfermedades emergentes, reemergentes y fronterizas. Epidemiol Salud 1: 16-20.

9. Nava S, Venzal JM, Gonzalez Acuña $D A$, Martins TF, Guglielmone AA. 2017. Ticks of the southern cone of America: diagnosis, distribution and hosts with taxonomy, ecology and sanitary importance. Londres: Elservier. 372 p.

10. Nava S, Mangold A, Mastropaolo M, Venzal J, Fracassi N, Guglielmone A. 2011. Seasonal dynamics and hosts of Amblyomma triste (Acari: Ixodidade) in Argentina. Vet Parasitol 181: 301-308. doi: 10.1016/j.vetpar.2011.03.054

11. Parola P, Raoult D. 2001. Ticks and tickborne bacterial diseases in humans: an emerging infectious threat. Clin Infect Dis 32: 897-928. doi: $10.1086 / 319347$

12. Pfäffle M, Littwin N, Petney T. 2015. The relationship between biodiversity and disease transmission risk. Review. Res Rep Biodiv Studies 4: 9-20. doi: 10.2147/ RRBS.S52433 
13. Sirolli H, de Miguel A, Chaparro G. 2018. Diagnóstico de situación y recomendaciones para la implementación de la Reserva Ecológica Ciudad Universitaria - Costanera Norte. Argentoina: Univ. de Buenos Aires. [Internet]. Disponible en: https://exactas.uba.ar/ reservaecologica-cu/informe-reservaecologica-CU.pdf

14. Soler-Tovar D, Romero-Prada JR, Villamil-Jiménez LC, Gómez-Ramírez AP, Jaimes-Olaya JA. 2010. Interfaces humano-animal-ecosistema: aproximación conceptual. Una 